\title{
1 Structure and lipid dynamics in the A. baumannii maintenance of lipid asymmetry (MLA) inner membrane complex
}

*Correspondence to: julien.bergeron@kcl.ac.uk

Daniel Mann ${ }^{1, \&}$, Junping Fan ${ }^{3, \%}$, Daniel P. Farrell ${ }^{4}$, Kamolrat Somboon ${ }^{5}$, Andrew Muenks ${ }^{4}$, Svetomir B. Tzokov ${ }^{1}$, Syma Khalid ${ }^{5}$,Frank Dimaio ${ }^{4}$, Samuel I. Miller ${ }^{3,4,6}$, Julien R. C. Bergeron ${ }^{1,2 *}$

${ }^{1}$ Department of Molecular Biology and Biotechnology, The University of Sheffield, Sheffield, United Kingdom

${ }^{2}$ Randall Division of Cell and Molecular Biophysics, King's College London, London, UK

${ }^{3}$ Department of Microbiology, The University of Washington, Seattle, USA

${ }^{4}$ Department of Biochemistry, The University of Washington, Seattle, USA

${ }^{5}$ Department of Chemistry, University of Southampton, Southampton, UK

${ }^{6}$ Department of Genetics, The University of Washington, Seattle, USA

\& Current address: Ernst-Ruska-Centre 3, Forschungszentrum Jülich, Germany

\% Current address: Department of Pharmacology, The University of Washington, Seattle, USA

Abstract

Multi-resistant bacteria are a major threat in modern medicine. The gram-negative coccobacillus Acinetobacter baumannii currently leads the WHO list of pathogens in critical need for new therapeutic development. The maintenance of lipid asymmetry (MLA) protein complex is one of the core machineries that transport lipids from/to the outer membrane in gram-negative bacteria. It also contributes to broad-range antibiotic resistance in several pathogens, most prominently in A. baumannii. Nonetheless, the molecular details of its role in lipid transport has remained largely elusive.

Here, we report the cryo-EM structures of the core MLA complex, MlaBDEF, from the pathogen A. baumannii, in the apo-, ATP- and ADB-bound states. These structures reveal multiple lipid binding sites, in the cytosolic and periplasmic side of the complex. Molecular dynamics simulations suggest their potential trajectory across the 
bioRxiv preprint doi: https://doi.org/10.1101/2020.05.30.125013; this version posted November $12,2020$. The copyright holder for this preprint (which was not certified by peer review) is the author/funder, who has granted bioRxiv a license to display the preprint in perpetuity. It is made available under aCC-BY-NC-ND 4.0 International license.

33 membrane. Collectively with the recently-reported structures of the E.coli orthologue, 34 these data also allows us to propose a molecular mechanism of lipid transport by the MLA system. 


\section{Introduction:}

Gram-negative bacteria are enveloped by two lipid bilayers, separated by the periplasmic space containing the peptidoglycan cell wall. The two membranes have distinct lipid compositions: The inner membrane (IM) consists of glycerophospholipids, with both leaflets having similar compositions, while the outer membrane (OM) is asymmetric, with an outer leaflet of lipopolysaccharides (LPS) and an inner leaflet of glycerophospholipids (1) (Fig. 1 A). This lipid gradient, depicting the first and most important permeation barrier is maintained by several machineries, including YebT, PqiB, and the multicomponent MLA system $(2,3)$, which consists of MlaA present in the OM, the shuttle MlaC in the periplasmic space, and the MlaBDEF ABC transporter system in the IM (Fig. $1 \mathrm{~A}$ ). The structure of some of these components have previously been solved: the OM protein MlaA, which was found to form a stable complex with outer membrane porins $\mathrm{OmpF}$ and $\operatorname{OmpC}(4,5)$, and the periplasmic protein MlaC revealing a hydrophobic pocket for direct lipid transport through the periplasm $(2,6)$. Low-resolution cryo-EM maps of the MlaBDEF core complex, from Escherichia coli $\left(\right.$ MlaBDEF $\left._{\mathrm{ec}}\right)(2)$ and Acinetobacter baumannii $\left(\right.$ MlaBDEF $\left._{\mathrm{ab}}\right)$ (7) have also been reported, and revealed the overall architecture of the complex, but did not allow to elucidate the molecular details of lipid binding and transport. Opinions about the directionality of lipid transport by the MLA system have been highly controversial, with initial reports suggesting that it recycles lipids from the $\mathrm{OM}$ to the $\operatorname{IM}(2,8,9)$, but recent results $(7,10,11)$ indicated that it might exports glycerophospholipids to the outer membrane.

In this study, we report the structure of the MlaBDEF ${ }_{a b}$ complex in detergent, in three nucleotide states, by single-particle cryo-EM. We also performed molecular dynamics simulations to gain insights into the dynamics of lipids within their observed binding sites. Collectively, this provides important new insights in the mechanism of lipid transport by the MLA system, and about the characterization of membrane proteins in detergent. 


\section{Results:}

\section{Structure of MlaBDEF $_{a b}$}

We had previously reported the purification of MlaBDEF $_{a b}$ in the presence of the detergent $n$-dodecyl $\beta$-D-maltoside (DDM), and its structure to $\sim 8 \AA$, by singleparticle cryo-EM, from data collected on a side-entry 200kV microscope (7). In order to improve the resolution of this structure, we collected a dataset of the same complex, in the presence of the non-hydrolizable ATP analogue App-NHp, using a state-of-the-art Titan Krios instrument. Using this better and larger dataset, we were able to refine the structure to $\sim 3.9 \AA$ resolution (Fig. 1B, Suppl. Fig. 1, Table 1). This map allowed us to build a de-novo atomic model using Rosetta (12) (See Materials and Methods for details).

As shown of figures $1 \mathrm{~B}$, and $1 \mathrm{C}$, the transmembrane multiprotein complex features a 6-fold symmetric assembly of MlaD, with the C-terminal helix forming a basket in the periplasmic space (Fig. 1D), and the N-terminal helix spanning the inner membrane (Fig. 1C). The N-terminal TM helices of MlaD are wrapped around the two MlaE molecules in the membrane, with three $\mathrm{MlaD}$ helices interacting asymmetrically with one MlaE, as reported previously (7). Intriguingly, while MlaE was predicted to have 6 TM helices, we observe that TM1 does not traverse the membrane, but is monotropically embedded in the inner leaflet, a feature similar to the G5G8 human sterol exporter (13), suggesting similar mechanisms between these complexes, and further supporting the MLA system as a lipid exporter. On the cytosolic side, MlaE is anchored into the ATPase MlaF via the coupling helix situated between TM3 and TM4 (Fig. 1), again similar to the G5G8 sterol exporter complex. MlaF is bound to MlaB away from the nucleotide binding site, similar to the recently-reported E.coli MlaBF structure (14), with C-termini of MlaF binding the opposing MlaB subunit by a "handshake" mode (Fig. 1E). We note, however, that particle classification demonstrated that only $\sim 50 \%$ of the particles included MlaB bound to both MlaF, leaving the other $50 \%$ bound to only one copy of MlaB (Suppl. Fig. 2). Dual binding of MlaB did not introduce major structural alterations at the detected resolution, and this observation may correspond to a regulatory role for MlaB or could be due to complex disassembly during sample preparation. 


\section{Lipids spontaneously bind into pockets of cytoplasmic MlaBDEF $\mathrm{ab}$}

In the cryo-EM derived map of MlaBDEF ${ }_{\mathrm{ab}}$, observed well-defined density in the pocket formed between the MlaE TM1, and two MlaD helices, within the inner leaflet of the lipid bilayer (Fig 2 A-C), which could not be interpreted by protein atoms. We also observed that this pocket is coated by cationic residues, mainly Arg14, Arg47 and Arg234 of MlaE, forming a charged pocket that might attract a lipid head group (Fig. 2D). This observation prompted us to propose that this density corresponds to detergent molecules bound to the complex. As the sample was solubilized in DDM, this density is most likely occupied by the maltoside ring, with the flexible hydrophobic chains extending up into an apolar region of MlaE. In order to confirm if the observed density was consistent with lipid positioning, we performed molecular dynamics simulations with unoccupied binding pockets as starting structures and observed rapid incorporation of bulk lipids during equilibration (Fig. 2 E). Newly bound lipids remained stably bound during 500 ns production runs (Fig. 2 F), depicting a reasonable first step in lipid export. This result is consistent with the position of lipid molecules within this region of the MlaBDEF ab $_{\text {complex. }}$

\section{Lipids bind into the periplasmic basket and partially flip}

The periplasmic region of MlaBDEF $_{a b}$ consists mainly of hexameric MlaD forming a basket shape (Fig. 1). Similar to other MCE domain proteins (15), MlaD consists of a central beta sheet motif with a central pore loop that is formed by hydrophobic Leu153/Leu154 in the center of the C6 symmetric complex (Fig. 3 A). We observed that in our map, unattributed density was present between the central pore loops, as well as in the central pore (Fig. 3 A). Importantly, this density is not an artifact of the 6fold symmetry, as it is also resolved when no symmetry was applied during the reconstruction. The presence of lipid molecules in very similar positions were previously reported in the MCE protein YebT (16), which prompted us to postulate that this unattributed density on the MlaD periplasmic region also corresponds to detergent molecules. Of note, the basket region has previously been proposed to form the binding site for the periplasmic carrier protein MlaC (4), suggesting that lipids are extracted from this position upon MlaC binding, consistent with the interpretation that these regions of the map correspond to lipid molecules. 
Because of this likely important role of lipid dynamics for this region, we next performed $3 \mathrm{D}$ variability analysis (17), to identify molecular motions in the MlaD crown. As shown on Suppl. movies 1 and 2, this analysis revealed that the 6x MlaD crown can be translated and rotated against the MlaBEF transmembrane part, which may play a role in the lipid transport mechanism. Incidentally, this dynamic property likely also limits the achievable resolution for this region of the map.

To further investigate the dynamic properties of the lipids present in the MlaD basket, we subjected them to molecular dynamics simulations. As shown on Figures $3 \mathrm{~B}-\mathrm{C}$, we observed that the peripheral lipids are rather stable during the course of the simulation. In contrast, the central lipid undergoes a significant motion within $150 \mathrm{~ns}$ of the trajectory. Remarkably, during this motion, we observed lipid flipping (Figure $3 \mathrm{D}$ ), with the head group which was modeled away from the central pore operating an almost $180^{\circ}$, with the polar group buried within the MlaE transporter. This observation likely indicates that we had initially build the lipid in the wrong orientation, and highlights the importance of properly modeling lipid molecules, especially at the intermediate resolution such as that of our MlaBDEF $_{a b}$ map. In addition, this demonstrates the presence of a large hydrophobic pocket at the MlaD-MlaE interface, which could correspond to the channel for lipid transport.

\section{Nucleotide binding occurs at the interface of MlaE, MlaF and MlaD}

As indicated above, our structure of MlaBDEF $\mathrm{ab}_{\mathrm{ab}}$ was determined in the presence of the non-hydrolizable ATP analogue AppNHp. Accordingly, we observed clear density of the nucleotide and Magnesium, within the MlaF binding pocket (Fig. 4A). The nucleotide links the ATPase subunits with the lipid transport domains at the interface of MlaF, MlaE and the N-terminus of MlaD. The resolution was sufficient to map the phosphate binding regions like Walker-A motif around Lys55 of MlaF, as well as binding of the $\gamma$-Phosphate by Ser51 and His211 ( $\mathrm{H}$-loop). The $\mathrm{Mg}^{2+}$-ion is coordinated by the Walker-B motif around Asp177 (Fig. 4A) and adenosine ring binding is achieved through Arg26 in the A-loop. As revealed in the previously-reported low-resolution map (7), the position of MlaF suggest that the complex is in the substrate-bound conformation. 
In order to identify structural changes in the complex associated with ATP binding and hydrolysis, we next determined the structure of MlaBDEF ${ }_{a b}$ without nucleotide (Suppl. Fig. 3), and with ADP (Suppl. Fig. 4). As shown on Figure 4A-C, we obtained both structures, to $\sim 4.2 \AA$ and $\sim 4.4 \mathrm{~A}$, respectively (Table 1 ). We note that in spite of the nominar global resolution, the map for the complex bound to ADP possesses features largely similar to that of the complex bound to AppNHp. In contrast, the map of the apo complex is less well resolved, in particular with most TM helices being mostly featureless. This suggests that nucleotide binding stabilizes the overall architecture of the MlaBDEF ab $_{\text {complex. }}$

Nonetheless, we note that the overlay of the MlaBDEF $_{a b}$ maps in the apo, AppNHpbound, and ADP-bound states show no major structural changes in any of the complex components, other than the nucleotide binding site (Fig. 4D). This indicates that in spite of the nucleotides being bound to the ATPase domain, under the conditions used this is not sufficient to trigger the activation of the channel opening.

\section{Discussion:}

While this manuscript was under review, three independent groups released the structure of the Escherichia coli MlaBDEF complex $\left(\right.$ MlaBDEF $_{\mathrm{ec}}$ ), in a range of nucleotide states and with various solubilization approaches (18-20).

Comparison of the MlaBDEF $_{\mathrm{ec}}$ structure (PDB-ID 7CH0, 7CGN), determined in lipid nanodiscs, and the MlaBDEF $_{\mathrm{ab}}$ structure (this study), determined in DDM, reveals a $\sim 10$ Ang movement of the MlaD TM helices, accompanied by a $\sim 30^{\circ}$ tilt of the MlaE $\mathrm{N}$-terminal helix (Fig. $5 \mathrm{~A}$ ). This movement narrows the cytoplasmic allocrit binding pocket significantly (Fig. $5 \mathrm{~A}$, red circles). A structural flexibility like this would allow adjustment of MlaBDEF for allocrits with varying sizes. However, as lateral pressure in the membrane plane of a detergent micelle is not comparable to a lipid nanodisc, differences in sample preparation can also cause movement of this hinge. Furthermore, although the MLA system is conserved in gram-negative bacteria, the sequence identity between the MlaBDEF $_{a b}$ MlaBDEF $_{\mathrm{ec}}$ proteins is around $30-40 \%$ identity (depending on the protein), and it is therefore possible that the differences observed between the two structures correspond to variability between bacterial species. 
Significantly, in one of the aforementioned studies, two ATP-bound conformational

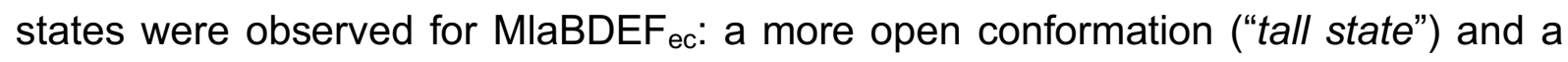
tightly bound conformation ("close state"), with a significantly shifted nucleotide position. The nucleotide position in MlaBDEF $_{\mathrm{ab}}$ in detergent resembles the tall state with an open conformation of MlaF (Fig. 5 B-C).

We also note that the lipid binding pockets observed in our MlaBDEF $a b$ structures are also confirmed in the MlaBDEF $\mathrm{ec}_{\mathrm{ec}}$ structures, notably the lipids found between the MlaD TM and the N-terminal helix of MlaE (20), and the lipid present in the central cavity (18-20). For the later, different exact lipid positions are observed depending on the state and study, but they are largely consistent with the lipid position obtained in the MD simulation, with the charged group buried at the MlaE-MlaD interface. In contrast, our map is the only one with clear lipid density at the interface between MlaD molecules in the periplasmic site; this could correspond to species specificity, or could be an artifact of the use of detergent for structure determination.

In the light of the multiple structures now available, the structures of MlaBDEF $\mathrm{ab}_{\mathrm{b}}$ reported here support a mechanism for lipid export as summarized in Figure 6. We observe lipids binding to the inner lipid binding pocket; this region is likely flexible and can possibly adapt to the size of the allocrit. MlaE is in very close proximity to ATP in the closed conformation, possibly describing an additional regulatory mechanism in $A$. baumannii. Structures of MlaBDEF $_{\mathrm{ec}}$ also showed a large-scale structural alteration upon tight ATP binding (20) that might be restrained due to the detergent environment in our experiments. We could furthermore observe a central lipid binding cite in the periplasmic basket region of MlaD that could partially flip into the central channel of MlaE during the course of our MD simulation. Nonetheless, neither our structures of MlaBDEF $_{\mathrm{ab}}$ nor the aforementioned MlaBDEF $_{\mathrm{ec}}$ could resolve allocrit transition between the cytoplasmic lipid binding pocket and the central channel. Similarly, allocrit transport from MlaBDEF to MlaC remains elusive.

It should be emphasized that the structural studies reported here support an anteretrograde direction for lipid transport, as supported by our previous biochemical data in $A$. baumannii (19) as well as other studies in $E$. coli $(11,19,20)$. Nonetheless, other assays point towards a retrograde directionality $(18,21)$, and the current structures do not conclusively preclude this. Further structural analyses, in particular 
in complex with the periplasmic carrier $\mathrm{MlaC}$, will be required to definitely resolve this controversy.

\section{Materials \& Methods:}

\section{MlaBDEF $_{\text {ab }}$ protein expression and purification}

The expression and purification of $\mathrm{MlaBDEF}_{\mathrm{ab}}$ has been described previously (7). Briefly, the plasmid was transformed into Escherichia coli BL21 DE3 cells and grown at $37^{\circ} \mathrm{C}$ until the cell density reached $\mathrm{OD}(600 \mathrm{~nm})=1.0$. The temperature was reduced to $20^{\circ} \mathrm{C}$ before induction with $1 \mathrm{mM}$ isopropyl $\beta$-D-thiogalactoside (IPTG) and incubation overnight. Cells were harvested using centrifugation at $5000 \times \mathrm{g}$ and resuspended in ice-cold buffer $\mathrm{A}(20 \mathrm{mM}$ Tris- $\mathrm{HCl}(\mathrm{pH} 8.0), 150 \mathrm{mM} \mathrm{NaCl}, 5 \%(\mathrm{v} / \mathrm{v})$ glycerol) before disruption in an ultrasonicator on ice (6 cycles; 60s run, 30s cooldown). Cell debris was centrifuged down at $17,000 \times \mathrm{g}$ for $10 \mathrm{~min}$ and the supernatant was centrifuged at $100,000 \times \mathrm{g}$ for $1 \mathrm{~h}$. This pellet was resuspended by gentle stirring in Buffer $\mathrm{A}$ supplemented with $1 \%(\mathrm{w} / \mathrm{v})$ dodecyl- $\beta$-d-maltopyranoside (DDM) at $7^{\circ} \mathrm{C}$ for $1 \mathrm{~h}$. After another centrifugation step at $100,000 \times \mathrm{g}$ for $30 \mathrm{~min}$ the supernatant was applied to a $5 \mathrm{ml}$ Ni-NTA superflow column (GE Healthsciences) that was previously equilibrated with $\mathrm{dd}_{2} \mathrm{O}$ and buffer $\mathrm{A}$ supplemented with $20 \mathrm{mM}$ imidazole and $0.025 \%$ (w/v) DDM. The column was washed with buffer A supplemented with $20 \mathrm{mM}$ imidazole and $0.025 \%(\mathrm{w} / \mathrm{v})$ DDM before elution with buffer A supplemented with $300 \mathrm{mM}$ imidazole and $0.025 \%(\mathrm{w} / \mathrm{v})$ DDM was performed. The elute was concentrated to $5 \mathrm{ml}$ and applied to a gel filtration run on a 16-600 HiLoad Superdex $200 \mathrm{pg}$ column (GE Healthcare), preequilibrated with $20 \mathrm{mM}$ Hepes (pH 7.0), $150 \mathrm{mM} \mathrm{NaCl}, 0.025 \%$ (w/v) DDM. Peak fractions were collected, purest fractions were selected using SDS-PAGE and concentrated to $5 \mathrm{mg} / \mathrm{ml}$.

\section{CryoEM sample preparation, data acquisition and image processing}

$4 \mu \mathrm{l}$ freshly purified $5 \mathrm{mg} / \mathrm{ml} \mathrm{MlaBDEF}_{\text {ab }}$ in $20 \mathrm{mM}$ Hepes $(\mathrm{pH} 7.0), 150 \mathrm{mM} \mathrm{NaCl}$, $0.025 \%$ (w/v) DDM was applied on 30s freshly glow discharged 300 mesh Quantifoil R2/2 grids, blotted for $3.5 \mathrm{~s}$ in a Leica EM-GP plunge freezer at $80 \%$ humidity and $4^{\circ} \mathrm{C}$, before getting plunged into liquid ethane at $-170^{\circ} \mathrm{C}$. For investigation of the $\mathrm{AppNHp}$ 
and ADP states, the protein was mixed 1:1 with $20 \mathrm{mM}$ of the corresponding nucleotide in $50 \mathrm{mM}$ Hepes (pH 8.0), $150 \mathrm{mM} \mathrm{NaCl}, 0.025 \%$ (w/v) DDM buffer and incubated for 60 min on ice prior to grid preparation.

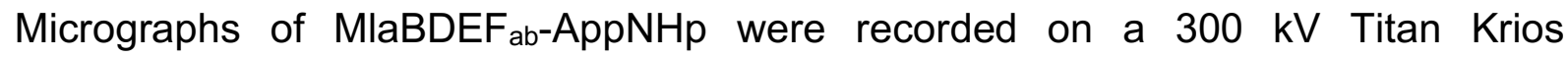
microscope with a Gatan K2 Summit detector in counting mode. 2557 movies were recorded with a pixel size of 1.07 Ang in 47 frames with 1e-/Ang²/frame. 1901 movies of MlaBDEF $\mathrm{ab}_{\mathrm{ab}}$-ADP were recorded on the same instrument with a total dose of $40 \mathrm{e}^{-}$ /Ang$^{2}$. The MlaBDEF $\mathrm{ab}$ apo dataset was recorded on a $300 \mathrm{KV}$ Titan Krios equipped with a Gatan K3 bioquantum detector. 4737 micrographs with a pixel size of 0.41 Ang were recorded with a total dose of $40 \mathrm{e}^{-/} / \mathrm{Ang}^{2}$ in 50 frames. Data processing was performed in CryoSPARC v2.14.2 (see Suppl. Fig. 1 and 3 for details). Raw images of

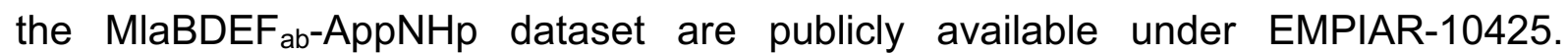
Sharpened maps in C2, C1 and C6 symmetry, masks and half-maps of MlaBDEF $\mathrm{ab}^{-}$ AppNHp, ADP and apo are publicly available under EMD-11082, EMD-11083 and EMD-11084, respectively.

\section{Model building}

The structural model of MlaBDEF $\mathrm{ab}_{\mathrm{ab}}$ was mally built into the high-resolved regions of the MlaBDEF ${ }_{a b}-A p p N H p$ map using Coot. Computational intervention was required to build residues 4-38, and 94-134 of MlaD, as well as refine the AppNHp binding site of MlaF. RosettaES (12) was used to model residues 4-38 for each of the $6 \mathrm{MlaD}$ subunits using the manually built model as the starting point. Residues 94-134 were first modeled with Rosetta Ab-initio (22) which yielded a tightly converged ensemble with a 2-helix topology. The top scoring model from the Ab-initio predictions was unambiguously docked into the corresponding density for each of the MlaD subunits using UCSF Chimera (23). Loops were completed and the entire MlaD crown refined using RosettaCM (24) with the context of the cryoEM density. To refine the AppNHp binding site of MlaF we first used RosettaCM to hybridize the manually built model with the homologous structures of ( $3 f v q$, chain $A)$, and (4ki0, chain $B$ ) in the cryoEM density. Then using homology models and the unexplained density as a guide, a modified version of the rosetta protocol GALigandDock (unpublished) that uses the cryoEM data to drive sampling was used to dock the AppNHp molecule. As input to the protocol, a mol2 file of AppNHp was modified using openbabel (v. 2.4.1) (25) to add hydrogens, 
charges were assigned with the AM1-BCC charge method in antechamber (26) and a params

file

was

generated

with

the

script

main/source/scripts/python/public/generic_potential/mol2genparams.py which is

distributed with Rosetta. Finally, the entire complex was refined using RosettaCM, and Magnesium atoms were added by incorporating distance and angle constraints between the $\mathrm{Mg}$ atoms and the AppNHp oxygens on the $\beta$ and $\mathrm{\gamma}$ phosphates during the final minimization. ISOLDE in ChimeraX was used to manually correct for modeling errors. Phenix.real.space.refine was used for refinement and validation. As shown on Suppl. Fig. 5 and 6, the resoluting model has an excellent fit to the map density, with clear fit of the side-chains in particular for the TM helices of MlaE and MlaD, which were critical for determining their registry. We note nonetheless that the TM for one of the MlaD molecules is mostly featureless (orange in suppl. Fig. 6), in which case we relied on the other two copies to position the helix in the density. The MlaBDEF ${ }_{a b}$ AppNHp model is publicly available under PDB-6Z5U.

\section{Molecular dynamics system preparation}

The MlaBDEF $_{a b}$ structure was completed by adding the missing residues using Modeller 9.23 (http://salilab.org/modeller/)(27, 28). A system, having the completed protein structure embedded in an inner E.coli innermembrane, was generated with the CHARMM GUI web interface(29-35) to have dimension of approximately $21 \times 21 \times$ $18 \mathrm{~nm}$ in $x y z$ dimension. The innermembrane composition was based on previous atomistic simulations studies(36):

$75 \%$ 1-palmitoyl-2-oleoyl-sn-glycero-3phosphoethanolamine (POPE), $20 \%$ 1-palmitoyl-2-oleoyl-sn-glycero-3phosphoglycerol (POPG) and 5\% 1',3'-bis[1,2-dioleoyl-sn-glycerol-3-phospho-]-snglycerol (Cardiolipin). To relax any steric conflicts within the system generated during set up, energy minimization of 5000 steps was performed on the starting conformation using the steepest descent method. An equilibration procedure followed in which the protein was subjected to position restraints with different force constant. The full equilibration protocol is shown in Suppl. Table 1. Fatty acid molecules were placed around the entrance of MLA using Visual Molecular Dynamics (VMD)(37). 
321 All simulations were carried out with GROMACS 2019.6(38) version

322 (www.gromacs.org) and CHARMM36(39) forcefield. For the nonbonded interactions

323 and the short-range electrostatics cut offs of $1.2 \mathrm{~nm}$ was applied to the system with the

324 potential shift Verlet cut off scheme, whereas the long-range electrostatic interactions were treated using the Particle Mesh Ewald (PME) method(40). All atoms were constrained using the LINCS algorithm(41) to allow the time step of 2 fs. The desired

327 temperature at either $310 \mathrm{~K}$ or $323 \mathrm{~K}$ was controlled with Nose-hoover thermostat(42, 328 43) (1.0 ps coupling constant). The pressure was maintained at $1 \mathrm{~atm}$ using the Parrinello-Rahman(44) semi-isotropic barostat with a coupling constant of 1.0 ps.

330 Equilibrium MD systems were performed with two repeats. Each repeat was run with different initial velocities. The summary of all production runs is shown in Suppl. Table 2. The results were analysed using built-in function in GROMACS package. Molecular graphic pictures were prepared using VMD. 


\section{Acknowledgments:}

338 We are thankful for financial support through BBSRC project BB/R019061/1. We 339 acknowledge Diamond Light Source for access and support of the cryo-EM facilities at 340 the UK's national Electron Bio-imaging Centre (eBIC) [under proposal EM-19832]. We 341 thank Emma Hasketh and Rebecca Thompson from the Astbury Centre for Structural 342 Molecular Biology Leeds for support during measurements. We acknowledge support 343 from LonCEM, King's College London during measurements. The University of 344 Sheffield FoS cryo-EM facility was used for grid preparation and optimization. We are 345 grateful to Justin Kollman for help with the initial stages of this project. 
1. Zhang, Y.-M., and Rock, C. O. (2008) Membrane lipid homeostasis in bacteria. Nat. Rev. Microbiol. 6, 222-233

350 2. Ekiert, D. C., Bhabha, G., Isom, G. L., Greenan, G., Ovchinnikov, S., Henderson, I. R., Cox, J. S., and Vale, R. D. (2017) Architectures of Lipid Transport Systems for the Bacterial Outer Membrane. Cell. 169, 273-285.e17

3. Isom, G. L., Coudray, N., MacRae, M. R., McManus, C. T., Ekiert, D. C., and Bhabha, G. (2020) LetB Structure Reveals a Tunnel for Lipid Transport across the Bacterial Envelope. Cell. 181, 653-664.e19

4. Abellón-Ruiz, J., Kaptan, S. S., Baslé, A., Claudi, B., Bumann, D., Kleinekathöfer, U., and van den Berg, B. (2017) Structural basis for maintenance of bacterial outer membrane lipid asymmetry. Nat. Microbiol. 2, 1616-1623

5. Yeow, J., Tan, K. W., Holdbrook, D. A., Chong, Z.-S., Marzinek, J. K., Bond, P. J., and Chng, S.-S. (2018) The architecture of the OmpC-MlaA complex sheds light on the maintenance of outer membrane lipid asymmetry in Escherichia coli. J. Biol. Chem. 293, 11325-11340

6. Huang, Y.-M. M., Miao, Y., Munguia, J., Lin, L., Nizet, V., and McCammon, J. A. (2016) Molecular dynamic study of MlaC protein in Gram-negative bacteria: conformational flexibility, solvent effect and protein-phospholipid binding. Protein Sci. Publ. Protein Soc. 25, 1430-1437

7. Kamischke, C., Fan, J., Bergeron, J., Kulasekara, H. D., Dalebroux, Z. D., Burrell, A., Kollman, J. M., and Miller, S. I. (2019) The Acinetobacter baumannii Mla system and glycerophospholipid transport to the outer membrane. eLife. 8, e40171

8. Malinverni, J. C., and Silhavy, T. J. (2009) An ABC transport system that maintains lipid asymmetry in the gram-negative outer membrane. Proc. Natl. Acad. Sci. U. S. A. 106, 8009-8014

9. Thong, S., Ercan, B., Torta, F., Fong, Z. Y., Wong, H. Y. A., Wenk, M. R., and Chng, S.-S. (2016) Defining key roles for auxiliary proteins in an ABC transporter that maintains bacterial outer membrane lipid asymmetry. eLife. 5, e19042

10. Hughes, G. W., Hall, S. C. L., Laxton, C. S., Sridhar, P., Mahadi, A. H., Hatton, C., Piggot, T. J., Wotherspoon, P. J., Leney, A. C., Ward, D. G., Jamshad, M., Spana, V., Cadby, I. T., Harding, C., Isom, G. L., Bryant, J. A., Parr, R. J., Yakub, Y., Jeeves, M., Huber, D., Henderson, I. R., Clifton, L. A., Lovering, A. L., and Knowles, T. J. (2019) Evidence for phospholipid export from the bacterial inner membrane by the Mla ABC transport system. Nat. Microbiol. 4, 1692-1705

11. Hughes, G. W., Sridhar, P., Nestorow, S. A., Wotherspoon, P. J., Cooper, B. phospholipid transport towards the outer membrane of Gram-negative bacteria. bioRxiv. 10.1101/2020.06.06.138008 
maps. Nat. Methods. 14, 797-800

13. Lee, J.-Y., Kinch, L. N., Borek, D. M., Wang, J., Wang, J., Urbatsch, I. L., Xie, X.-S., Grishin, N. V., Cohen, J. C., Otwinowski, Z., Hobbs, H. H., and Rosenbaum, D. M. (2016) Crystal structure of the human sterol transporter ABCG5/ABCG8. Nature. 533, 561-564

14. Kolich, L., Chang, Y-T., Coudray, N., Giacometti, S. I., MacRae, M. R., Isom, G. L., Teran, E. M., Bhabha, G., and Ekiert, D. C. (2020) Structure of MlaFB uncovers novel mechanisms of $\mathrm{ABC}$ transporter regulation. bioRxiv 10.1101/2020.04.27.064196v1.full

15. Isom, G. L., Davies, N. J., Chong, Z.-S., Bryant, J. A., Jamshad, M., Sharif, M., Cunningham, A. F., Knowles, T. J., Chng, S.-S., Cole, J. A., and Henderson, I. R. (2017) MCE domain proteins: conserved inner membrane lipid-binding proteins required for outer membrane homeostasis. Sci. Rep. 7, 1-12

16. Liu, C., Ma, J., Wang, J., Wang, H., and Zhang, L. (2020) Cryo-EM Structure of a Bacterial Lipid Transporter YebT. J. Mol. Biol. 432, 1008-1019

17. Punjani, A., and Fleet, D. J. (2020) 3D Variability Analysis: Directly resolving continuous flexibility and discrete heterogeneity from single particle cryo-EM images. bioRxiv. 10.1101/2020.04.08.032466

18. Tang, X., Chang, S., Qiao, W., Luo, Q., Chen, Y., Jia, Z., Coleman, J., Zhang, K., Wang, T., Zhang, Z., Zhang, C., Zhu, X., Wei, X., Dong, C., Zhang, X., and Dong, H. (2020) Structural insight into outer membrane asymmetry maintenance of Gramnegative bacteria by the phospholipid transporter MlaFEDB. bioRxiv. 10.1101/2020.06.04.133611

19. Coudray, N., Isom, G. L., MacRae, M. R., Saiduddin, M. N., Bhabha, G., and Ekiert, D. C. (2020) Structure of bacterial phospholipid transporter MlaFEDB with substrate bound, BioRXiv, 10.1101/2020.06.02.129247

20. Chi, X., Fan, Q., Zhang, Y., Liang, K., Wan, L., Zhou, Q., and Li, Y. (2020) Structural mechanism of phospholipids translocation by MlaFEDB complex. Cell Res. 10.1038/s41422-020-00404-6

21. Powers, M. J., Simpson, B. W., and Trent, M. S. (2020) The Mla pathway in Acinetobacter baumannii has no demonstrable role in anterograde lipid transport. eLife. 9, e56571

22. Simons, K. T., Bonneau, R., Ruczinski, I., and Baker, D. (1999) Ab initio protein structure prediction of CASP III targets using ROSETTA. Proteins Struct. Funct. Bioinforma. 37, 171-176

23. Pettersen, E. F., Goddard, T. D., Huang, C. C., Couch, G. S., Greenblatt, D. M., Meng, E. C., and Ferrin, T. E. (2004) UCSF Chimera--a visualization system for exploratory research and analysis. J. Comput. Chem. 25, 1605-1612

24. Song, Y., DiMaio, F., Wang, R. Y.-R., Kim, D., Miles, C., Brunette, T., Thompson, J., and Baker, D. (2013) High resolution comparative modeling with RosettaCM. Struct. Lond. Engl. 1993. 10.1016/j.str.2013.08.005 
25. O’Boyle, N. M., Banck, M., James, C. A., Morley, C., Vandermeersch, T., and Hutchison, G. R. (2011) Open Babel: An open chemical toolbox. J. Cheminformatics. 3, 33

26. Wang, J., Wang, W., Kollman, P. A., and Case, D. A. (2006) Automatic atom type and bond type perception in molecular mechanical calculations. J. Mol. Graph. Model. 25, 247-260

27. Šali, A., and Blundell, T. L. (1993) Comparative Protein Modelling by Satisfaction of Spatial Restraints. J. Mol. Biol. 234, 779-815

28. Sánchez, R., and Šali, A. (2000) Comparative Protein Structure Modeling: Introduction and Practical Examples with Modeller. in Protein Structure Prediction, pp. 97-129, Humana Press, New Jersey, 10.1385/1-59259-368-2:97

29. Jo, S., Kim, T., Iyer, V. G., and Im, W. (2008) CHARMM-GUI: A web-based graphical user interface for CHARMM. J. Comput. Chem. 29, 1859-1865

30. Brooks, B. R., Brooks, C. L., Mackerell, A. D., Nilsson, L., Petrella, R. J., Roux, B., Won, Y., Archontis, G., Bartels, C., Boresch, S., Caflisch, A., Caves, L., Cui, Q., Dinner, A. R., Feig, M., Fischer, S., Gao, J., Hodoscek, M., Im, W., Kuczera, K., Lazaridis, T., Ma, J., Ovchinnikov, V., Paci, E., Pastor, R. W., Post, C. B., Pu, J. Z., Schaefer, M., Tidor, B., Venable, R. M., Woodcock, H. L., Wu, X., Yang, W., York, D. M., and Karplus, M. (2009) CHARMM: The biomolecular simulation program. J. Comput. Chem. 30, 1545-1614

31. Lee, J., Cheng, X., Swails, J. M., Yeom, M. S., Eastman, P. K., Lemkul, J. A., Wei, S., Buckner, J., Jeong, J. C., Qi, Y., Jo, S., Pande, V. S., Case, D. A., Brooks, C. L., MacKerell, A. D., Klauda, J. B., and Im, W. (2016) CHARMM-GUI Input Generator for NAMD, GROMACS, AMBER, OpenMM, and CHARMM/OpenMM Simulations Using the CHARMM36 Additive Force Field. J. Chem. Theory Comput. 12, 405-413

32. Wu, E. L., Cheng, X., Jo, S., Rui, H., Song, K. C., Dávila-Contreras, E. M., Qi, Y., Lee, J., Monje-Galvan, V., Venable, R. M., Klauda, J. B., and Im, W. (2014) CHARMM-GUI Membrane Builder toward realistic biological membrane simulations. J. Comput. Chem. 35, 1997-2004

33. Jo, S., Lim, J. B., Klauda, J. B., and Im, W. (2009) CHARMM-GUI membrane builder for mixed bilayers and its application to yeast membranes. Biophys. J. 97, 5058

34. Jo, S., Kim, T., and Im, W. (2007) Automated Builder and Database of Protein/Membrane Complexes for Molecular Dynamics Simulations. PLoS ONE. 2, e880

35. Lee, J., Patel, D. S., Ståhle, J., Park, S. J., Kern, N. R., Kim, S., Lee, J., Cheng, X., Valvano, M. A., Holst, O., Knirel, Y. A., Qi, Y., Jo, S., Klauda, J. B., Widmalm, G., and Im, W. (2019) CHARMM-GUI Membrane Builder for Complex Biological Membrane Simulations with Glycolipids and Lipoglycans. J. Chem. Theory Comput. 15, 775-786

36. Holdbrook, D. A., Huber, R. G., Piggot, T. J., Bond, P. J., and Khalid, S. (2016) 
471 Dynamics of Crowded Vesicles: Local and Global Responses to Membrane

472 Composition. PLOS ONE. 11, e0156963

473 37. Humphrey, W., Dalke, A., and Schulten, K. (1996) VMD: visual molecular

474 dynamics. J. Mol. Graph. 14, 33-8, 27-8

475 38. Van Der Spoel, D., Lindahl, E., Hess, B., Groenhof, G., Mark, A. E., and

476 Berendsen, H. J. C. (2005) GROMACS: Fast, flexible, and free. J. Comput. Chem.

$477 \quad 26,1701-1718$

478 39. Huang, J., and MacKerell, A. D. (2013) CHARMM36 all-atom additive protein

479 force field: Validation based on comparison to NMR data. J. Comput. Chem. 34,

$480 \quad 2135-2145$

481 40. Essmann, U., Perera, L., Berkowitz, M. L., Darden, T., Lee, H., and Pedersen,

482 L. G. (1995) A smooth particle mesh Ewald method. J. Chem. Phys. 103, 8577-8593

483 41. Hess, B., Bekker, H., Berendsen, H. J. C., and Fraaije, J. G. E. M. (1997)

484 LINCS: A linear constraint solver for molecular simulations. J. Comput. Chem. 18,

$485 \quad 1463-1472$

486 42. Hoover, W. G. (1985) Canonical dynamics: Equilibrium phase-space

487 distributions

488 43. Nosé, S. (1984) A molecular dynamics method for simulations in the canonical 489 ensemble. Mol. Phys. 52, 255-268

490 44. Parrinello, M., and Rahman, A. (1981) Polymorphic transitions in single

491 crystals: A new molecular dynamics method. J. Appl. Phys. 52, 7182-7190 


\section{A}
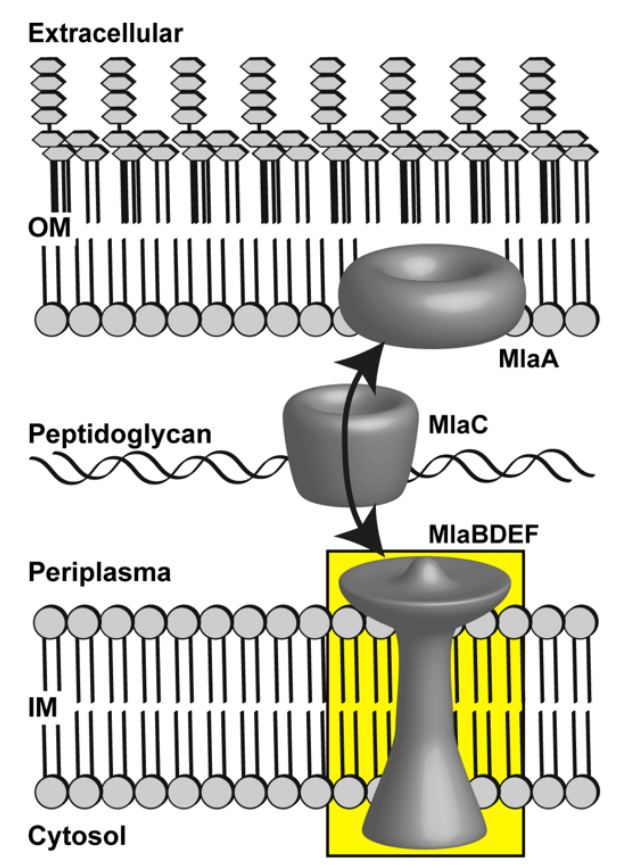

B
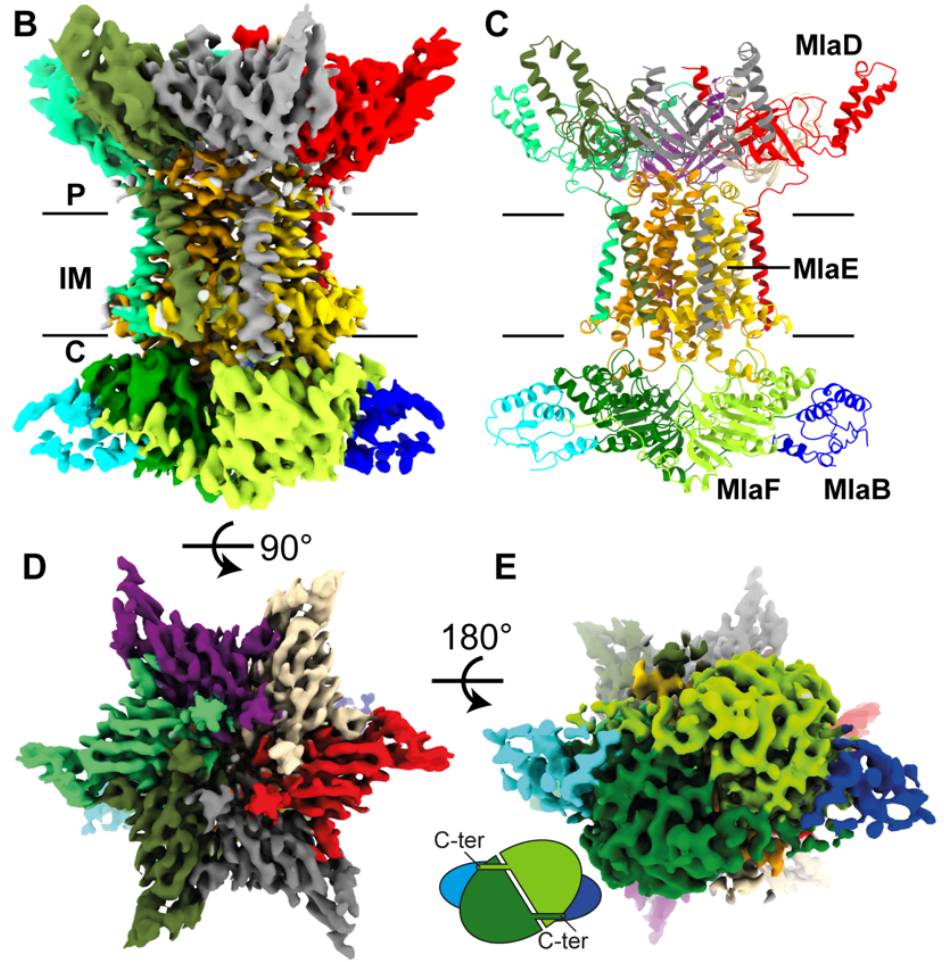

Figure 1: Structure of MlaBDEF Mb $_{\text {ab }}$ Scheme of lipid transport by the MlaABCDEF system in gram-negative bacteria (OM = outer membrane; $\mathrm{IM}=$ inner membrane). $\mathrm{B}$ : $3.9 \AA$ CryoEM map of MlaBDEF ${ }_{a b}-A p p N H p$ in DDM (C=cytosol, IM=inner membrane, $\mathrm{P}=$ periplasma). C: MlaBDEF $\mathrm{ab}_{\mathrm{b}}$ has global $\mathrm{C} 2$ symmetry with six copies of MlaD that span the inner membrane from the periplasmic space to the cytosol, two copies of MlaE embedded in the membrane (yellow/gold), two copies of the ATPase MlaF in the cytosol (green), each bound to a copy of MlaB (blue/cyan). D: top view of the the opposing MlaB subunit via a "handshake-mechanism". 
A

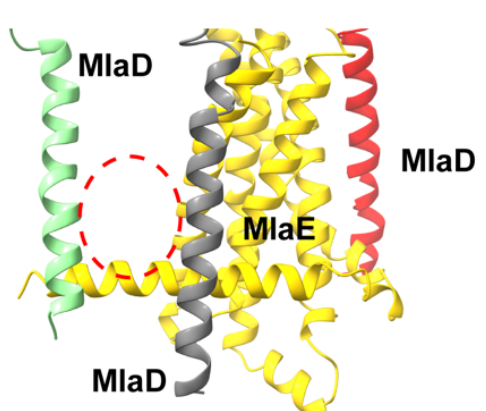

B

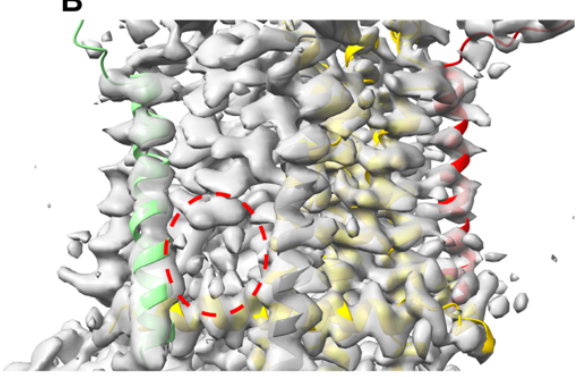

C

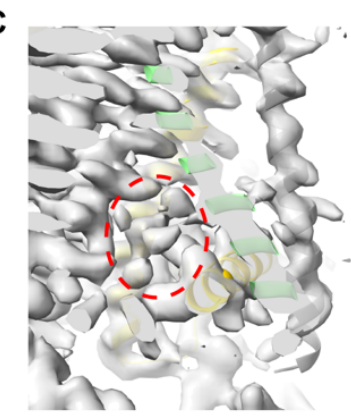

D

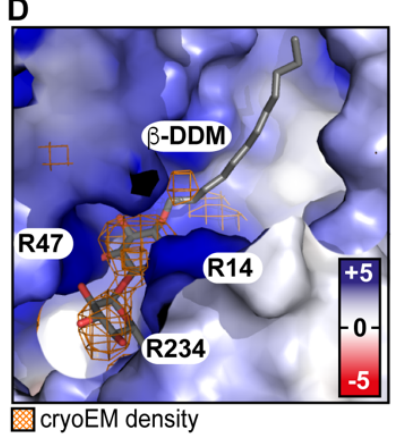

E

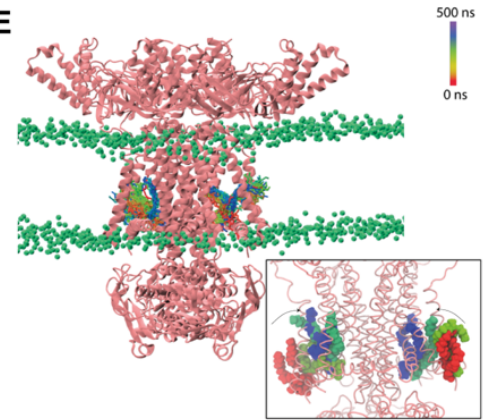

$\mathbf{F}$

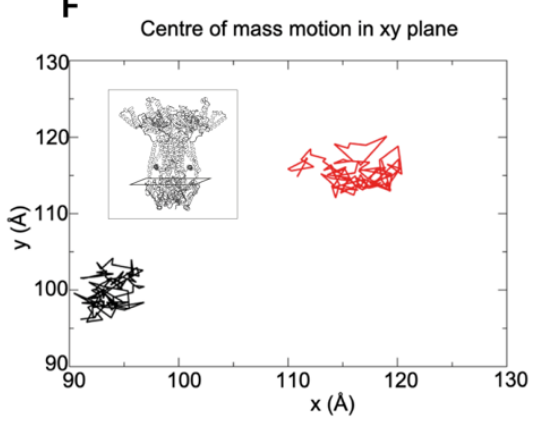

Figure 2: Detergent position and dynamics at the MlaD TM-MlaE interface. A-C:

Transmembrane region of MlaBDEF $\mathrm{ab}_{\mathrm{b}}$ with one MlaE and three MlaD proteins shown. Red circles indicates density unoccupied by protein residues. D: Electrostatic surface potential of modeled inner membrane MlaBDEF $_{a b}$ (blue $=+5 \mathrm{kT} / \mathrm{e}$, white $=0 \mathrm{kT} / \mathrm{e}$, red=$5 \mathrm{kT} / \mathrm{e}$ ) shows a cationic binding site for anionic lipid head groups like -DDM and an apolar pocket for the uncharged lipid tails. -DDM was modeled inside the previously empty CryoEM density (orange). E-F: Molecular dynamics simulations show spontaneous, stable binding of lipids into the cytosolic binding pockets of MlaBDEF. Lipid-free MlaBDEF $_{a b}$ was embedded into an inner membrane model system of $75 \%$ POPE, 20\% POPG, 5\% Cardiolipin (Methods section for details). Panel E shows the location of two POPE lipids during a 500 ns simulation, the colour scheme indicates the movement of the lipids as shown in the legend. In this simulation the lipids moved into this location spontaneously during the equilibration process, as shown in the closeup view in the inset in which the red coloured lipids indicate starting positions before the equilibration process. Panel $F$ shows the motion of the lipids in the xy plane (inset) over 500 ns. The lipids are confined within an area of $10 \times 10 \AA$ for 500 ns, showing this location is favourable. 
A

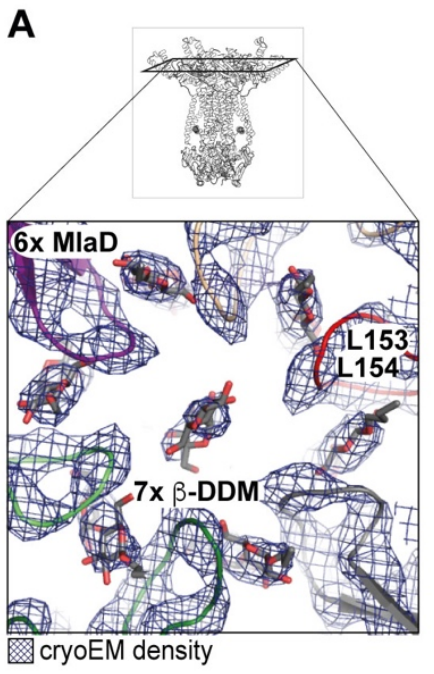

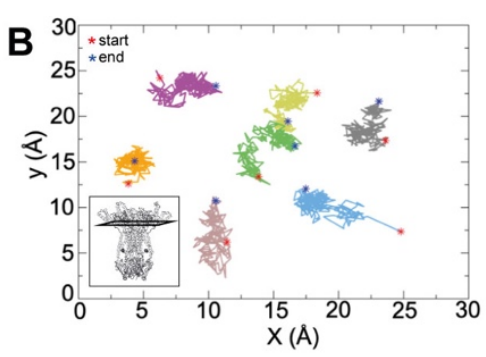

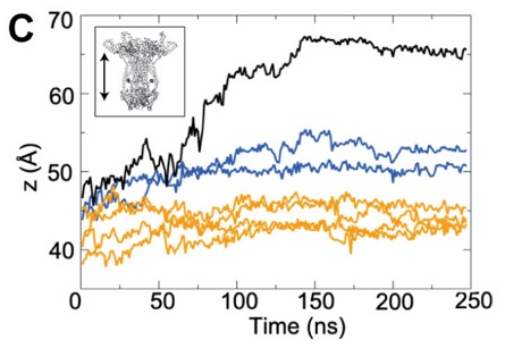

D
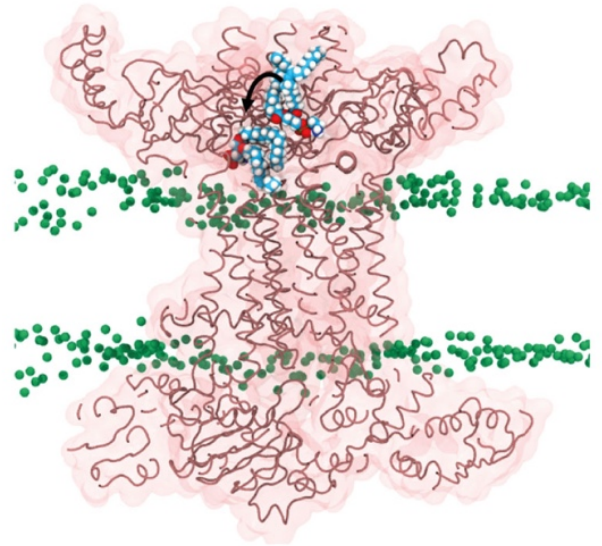

527

Figure 3: Lipid position and dynamics in the MlaD basket. A: Close-up of the periplasmic MlaD basket that houses six peripheral and one central detergent molecule. B-D: Spontaneous lipid flipping within the basket region of MlaBDEF $_{a b}$ during 250 ns molecular dynamics simulations. Panel B shows the center of mass motion of the seven lipids in the xy plane. They are confined to an area of $\sim 5 \times 5 \AA$, indicating this is a high lipid affinity region. Panel $\mathrm{C}$ shows the center of mass movement of the seven lipids in the $Z$ dimension as a function of time. The higher values of $z$ correspond to the cytoplasmic end of the protein. The lipid shown in panel A corresponds to the black curve showing a clear movement towards the cytoplasmic end. Three other lipids (blue) move into this channel but to a lesser extent than the aforementioned lipid, whereas three others (orange) remain close to their starting positions. Simulations from a model at the reported resolution cannot clarify the directionality of the movement, but rather that these regions are conduits for lipids. Panel D shows a cut-away view of the protein with a POPE lipid at two time points during the simulation, time $=0 \mathrm{~ns}$ and $150 \mathrm{~ns}$. The simulation was initiated with seven POPE lipids placed at the periplasmic end of the protein corresponding to the density for detergents in the cryo-EM data (Table S2). The lipid which is displaced more towards the cytoplasmic end is from the frame at $t=150 \mathrm{~ns}$. The central hydrophobic 'channel' of the protein is a clear conduit for lipids given the spontaneous movement of POPE into this region in just 150 ns. 


\section{A}

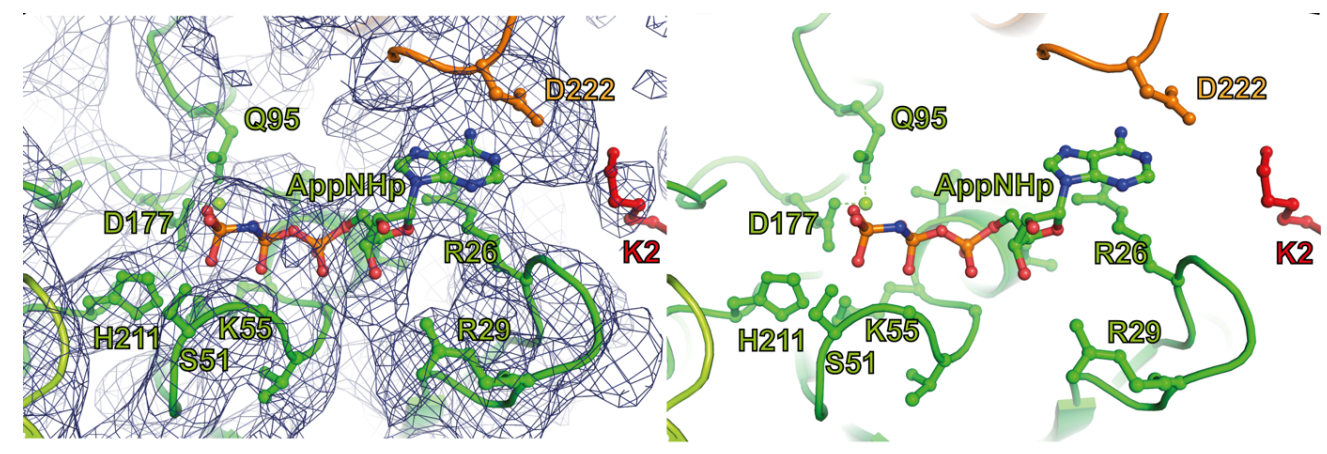

B

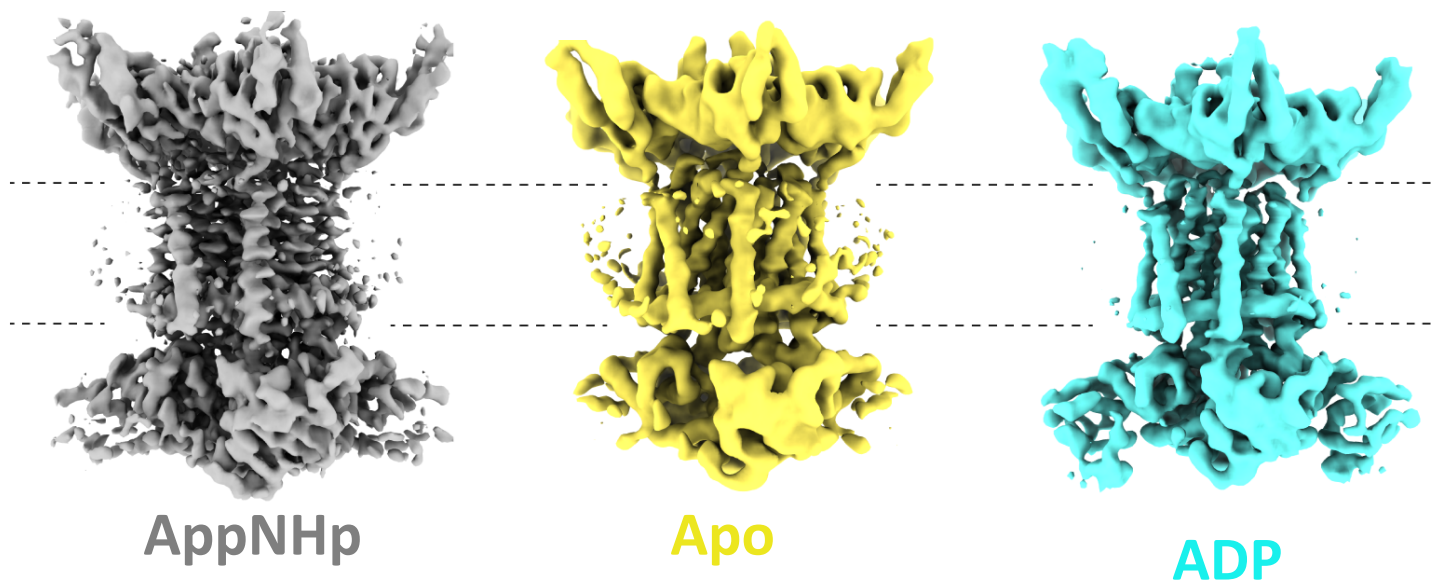

C
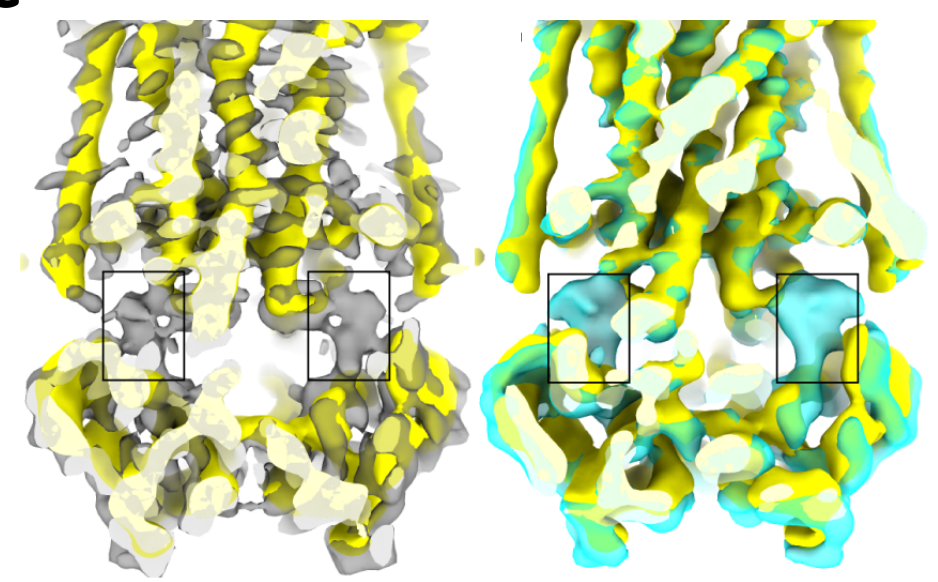

\section{AppNHp}

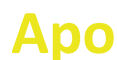

ADP

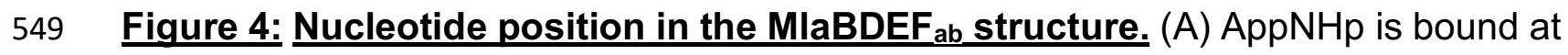
the interface of MlaE (orange), MlaD (red) and MlaF (green). B: maps of MlaBDEF ${ }_{a b}$ bound to AppNHp (grey), its apo state (yellow) and bound to ADP (cyan). (C) The overlay of the maps unambiguously confirms the presence of nucleotide in the AppNHp and ADP maps, but not in the Apo map. Nonetheless, no overall structural changes is observed. 

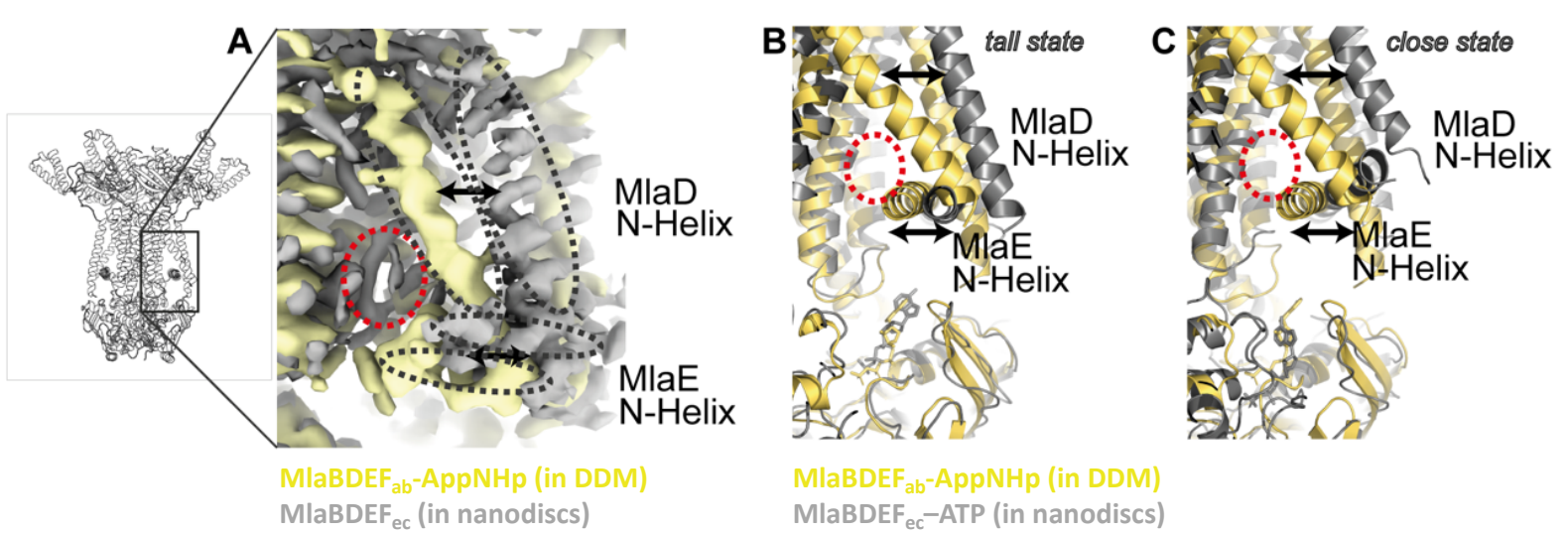

MlaBDEF ${ }_{\text {ab-AppNHp (in DDM) }}$

MlaBDEF $_{\mathrm{ec}}-\mathrm{ATP}$ (in nanodiscs)

557 Figure 5: Comparison of the MlaBDEF $_{\mathrm{ab}}$ and MlaBDEF $_{\mathrm{ec}}$ structures. Acquired

558 Cryo-EM map of MlaBDEF ec $_{\text {in lipid nanodiscs (20) and MlaBDEF }}$ ab in a DDM micelle 559 (this study) show large differences in the lower lipid binding sites. A: Both the MlaD N560 terminal helix and the MlaE N-terminal helix form a much smaller lipid binding pocket 561 (red circle) in detergent environment (yellow, this study) compared to lipid environment 562 (grey, EMD-30355). Two nucleotide binding states were observed in the MlaBDEF 563 study; "tall" and "close". Comparison to B) "tall state" (grey) shows almost identical 564 nucleotide position between MlaBDEF $_{\mathrm{ec}}$ and MlaBDEF $_{\mathrm{ab}}$, whereas 

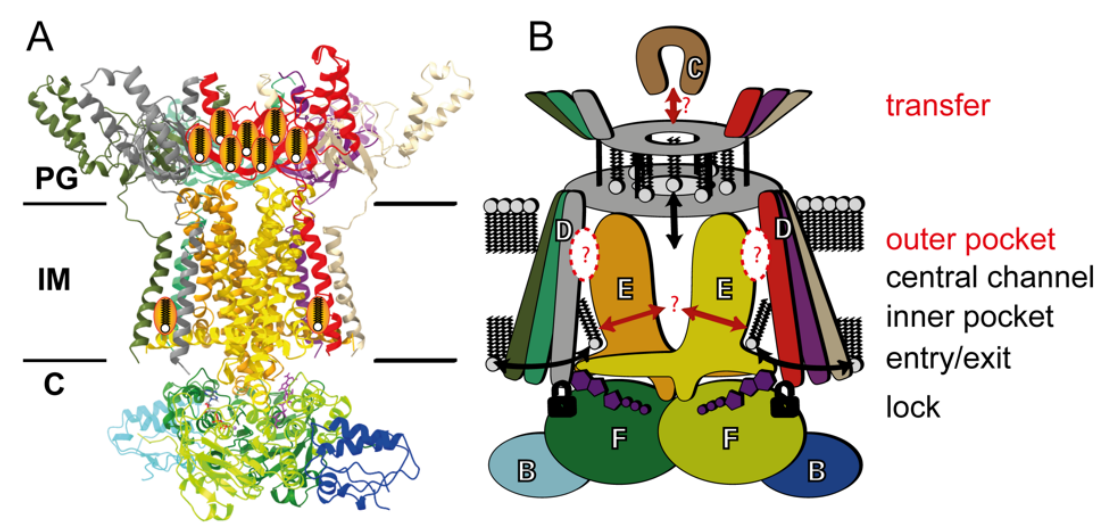

568 Figure 6: Molecular model of lipid transport by the MlaBDEF complex. A:

569 structural model and B: observed degrees of freedom in this study. Lipids can move

570 freely into the cytosolic paired binding pockets ("entry/exit") that are very close to the 571 ATP binding sites that directly bind MlaD ("lock"). Lipids can move and even flip inside

572 the basket region ("transport"). C: The elucidated structures could not show how 573 transport from the entry sites to the basket region is performed and how lipid exchange 574 to/from MlaC is achieved. 
Table 1: Cryo-EM data processing and refinement statistics

\begin{tabular}{|c|c|c|c|}
\hline & $\begin{array}{c}\text { AppNHp } \\
\text { (EMDB-11082 } \\
\text { (PDB: 6Z5U) }\end{array}$ & $\begin{array}{c}\text { Apo } \\
(\text { EMDB-11083) }\end{array}$ & $\begin{array}{c}\text { ADP } \\
(\text { EMDB-11084) }\end{array}$ \\
\hline \multicolumn{4}{|l|}{$\begin{array}{l}\text { Data Collection and } \\
\text { Processing }\end{array}$} \\
\hline Microscope & Titan Krios & Titan Krios & Titan Krios \\
\hline Voltage (kV) & 300 & 300 & 300 \\
\hline Camera & K2 summit & K3 bioquantum & K2 summit \\
\hline Pixel size $(\AA)$ & 1.07 & 0.41 & 1.07 \\
\hline Defocus range $(\mu \mathrm{m})$ & -1 to -2.5 & -0.8 to -2.0 & -1 to -2.5 \\
\hline Total dose $\left(\mathrm{e}^{\circ}{ }^{-2}\right)$ & 47 & 40 & 40 \\
\hline Number of micrographs & 2557 & 4737 & 1901 \\
\hline Total particles used & 93,295 & 28,699 & 62,512 \\
\hline Map Resolution $(\AA)$ & 3.92 & 4.24 & 4.43 \\
\hline \multicolumn{4}{|l|}{ Refinement } \\
\hline \multicolumn{4}{|l|}{ Model composition } \\
\hline Non-hydrogen atoms & 17,892 & & \\
\hline Protein Residues & 2334 & & \\
\hline Ligand atoms & 4 & & \\
\hline \multicolumn{4}{|l|}{$B$ factors $\left(\AA^{-1}\right)$} \\
\hline Protein & 145.24 & & \\
\hline Ligand & 170.31 & & \\
\hline \multicolumn{4}{|l|}{ R.m.s. deviations } \\
\hline Bond lengths $(\AA)$ & 0.008 & & \\
\hline Bond angles $\left(^{\circ}\right)$ & 1.384 & & \\
\hline \multicolumn{4}{|l|}{ Validation } \\
\hline MolProbity score & 3.43 & & \\
\hline Clashscore & 35.15 & & \\
\hline Poor rotamers (\%) & 10.94 & & \\
\hline \multicolumn{4}{|l|}{ Ramachandran plot } \\
\hline Favoured (\%) & 87.45 & & \\
\hline Allowed (\%) & 10.94 & & \\
\hline Disallowed (\%) & 0.43 & & \\
\hline
\end{tabular}

